\title{
Cinquentenário da Simulação Computacional em Mecânica Estatística
}

\author{
I. Os primeiros passos
}

\author{
FER N A D O M. S. SILVA FER N A N D E S *
}

\section{Introdução}

Em Junho de 2003 passaram cinquenta anos desde a publicação, no Journal of Chemical Physics, do célebre artigo "Equation of State Calculations by Fast Computing Machines" da autoria de Nicholas Metropolis, Arianna e Marshall Rosenbluth, e Augusta e Edward Teller [1]. Este trabalho marca, historicamente, a entrada definitiva do método de Monte Carlo e da simulação computacional na mecânica estatística.

Durante os cinquenta anos da sua existência, a simulação computacional estabeleceu-se como um domínio interdisciplinar em estreita ligação com diversas áreas teóricas e experimentais. Considerando o desenvolvimento do conhecimento, cada vez mais refinado, dos princípios científicos fundamentais e a produção acelerada de hardware e software computacionais, pode afirmar-se que a simulação computacional é um domínio do futuro.

É justo, por conseguinte, que se assinale o meio-século de vida da simulação computacional em mecânica estatística, recordando o seu nascimento, desenvolvimento e as aplicações mais relevantes. Tanto mais que, em Portugal, existem grupos de investigação que a utilizam e desenvolvem, ela faz parte dos curricula de licenciaturas e mestrados, e são realizados doutoramentos nesse domínio.

Nos Estados Unidos, em Los Alamos [2], o berço do nascimento do método de Monte Carlo, a efeméride foi comemorada com uma Conferência Interna- cional em Junho de 2003. A Divisão de Química-Física, da Sociedade Portuguesa de Química, promoverá um Colóquio sobre o tema durante 2003.

Neste artigo, descrevemos os primeiros passos do método de Monte Carlo. Num próximo artigo, abordaremos o desenvolvimento e perspectivaremos o futuro da simulação computacional.

\section{O nascimento}

A simulação computacional é, naturalmente, uma consequência directa da construção dos primeiros computadores electrónicos na década de 1940-50. Neste contexto, o melhor representante é o ENIAC (Electronic Numerical Integrator And Computer) construido em 1946 por J.P. Eckert e J.W. Mauchly na Universidade da Pensilvânia, EUA. Foi nesta máquina que von Neumann, Ulam, Metropolis e Frankel realizaram alguns cálculos numéricos estocásticos sobre a difusão de neutrões em processos nucleares. Numa conferência em Los Alamos, Abril de 1946, eles descreveram as potencialidades da máquina e Ulam sugeriu que a velocidade de cálculo desses equipamentos poderia possibilitar o uso, em larga-escala, de técnicas de amostragem estatística para a resolução de problemas físicos e matemáticos, as quais não eram mais do que as precursoras do actual método de Monte Carlo.

A ideia, contudo, era mais velha! Na verdade, Segrè [3] relata: "Eu sei que Fermi tinha inventado, mas não designado o método de Monte Carlo quando, ainda em Roma, investigava a moderação de neutrões. Nada publicou sobre o assunto, mas usou o método para resolver muitos problemas recorrendo a quaisquer instrumentos de cálculo disponíveis, em particular uma pequena máquina de somar mecânica". Curiosamente, em 1947, enquanto o ENIAC esteve desactivado, durante a sua transferência para a Universidade de Aberdeen, Maryland, Enrico Fermi inventou e construiu com $\mathrm{P}$. King um pequeno computador analógico para realizar cálculos sobre a difusão de neutrōes [4].

Estes factos merecem ser recordados, não só pelo seu valor histórico, mas também porque contrastam com uma atitude actual generalizada, particularmente das pessoas mais novas, no que diz respeito ao material informático. 0 espectacular desenvolvimento de hardware e software e a constante descida de preços, convida a que se desprezem, da noite para o dia, máquinas em condições ainda óptimas para a realização de cálculos científicos. Máquinas que teriam feito as delícias de Enrico Fermi! Quantos "cemitérios" de material informático se evitariam se esses processadores fossem utilizados para constituir "clusters" de alto desempenho. E quantos resultados originais se produziriam, a baixo custo, desde o momento que não faltassem as ideias que os computadores não podem, de todo, formalizar.

Quanto ao nome "Monte Carlo" parece ter sido Metropolis [4] o primeiro a pensar nele numa conversa com Ulam, em 1947, sobre as técnicas estocásticas para o estudo da difusão de neutrões. 0 
nome surge, pela primeira vez, no título de um artigo de Metropolis e Ulam [5] em 1949.

Seria injusto, contudo, não destacar o papel crucial de von Neumann neste contexto. John von Neumann, nascido na Hungria, foi uma das mentes mais brilhantes e rápidas do século XX. Aos seis anos de idade dividia, mentalmente, números com oito algarismos e falava com o Pai em grego antigo. Aos vinte e seis anos lançou os fundamentos rigorosos da mecânica quântica, com base no conceito de espaço de Hilbert [6]. No início dos anos 30 estabeleceu-se definitivamente nos Estados Unidos e foi, juntamente com Albert Einstein, um dos primeiros residentes do reputado Instituto de Estudos Avançados de Princeton, criado em 1933. As suas contribuições estendem-se desde a matemática e física até à ciência dos computadores, robótica e autómatos celulares. Foi ele que implementou a ideia de "programa residente", isto é, a máquina ser controlada por software interno e não, como no caso do ENIAC, por um processo externo e complicado de ligações por hardware. Embora a origem da ideia seja obscura (não se sabe exactamente se foi dele, dos construtores do ENIAC, Eckert e Mauchly, ou do matemático inglês Alan Turing, o qual introduziu o conceito de "máquina de Turing", a base formal dos computadores) parece não restarem dúvidas que foi von Neumann que a efectivou num protótipo que construiu no Instituto de Princeton [7]. Tendo estado envolvido no projecto Manhattan, em Los Alamos, colaborou, como referimos, no estudo dos processos estocásticos, isto é, processos que evoluem no tempo sujeitos a flutuações aleatórias. Eles são, afinal, o cerne do método de Monte Carlo. Para os implementar numericamente é imprescindível um bom gerador de números aleatórios. John von Neumann foi pioneiro no seu desenvolvimento e implementação [8-10]

A ideia da amostragem estatística numérica tem, no entanto, uma origem ainda mais antiga [11]. Por exemplo, Lord Kelvin [12] utilizou técnicas de amostragem aleatória para inicializar trajectórias de partículas sujeitas a colisões elásticas com as paredes de recipientes de diferentes formas. Isto em relação à disputa, no início do século XX, sobre as falhas do princípio da equipartição de energia e os fundamentos da mecânica estatística. Lord Kelvin agradeceu ao seu secretário, William Andersen, por ter realizado os cálculos para mais de 5000 colisões!

Quando em Março de 1952, o célebre computador MANIAC (Mathematical
O espaço configuracional do sistema é representado por um conjunto com um número finito $(\mathrm{M}$ ) de configurações (estados), cada uma identificada pelas coordenadas de cada molécula. Por definição, uma propriedade mecânica, f, depende das coordenadas moleculares, portanto encontra-se definida para cada configuração do ensemble. A média canónica dessa propriedade sobre os $\mathrm{M}$ estados do ensemble é dada, de acordo com a mecânica estatística, por:

$\langle f\rangle=(1 / Q) \sum_{i=1}^{M} f(i) \exp [-\beta E(i)] \operatorname{com} Q=\sum_{i=1}^{M} \exp [-\beta E(i)]$

Analyser, Numerator, Integrator And Computer), um sucessor do ENIAC, entrou em funcionamento em Los Alamos, Nicholas Metropolis estava interessado em resolver um conjunto grande e variado de problemas de modo a avaliar a estrutura lógica da máquina e a demonstrar as suas capacidades. 0 clássico problema dos $\mathrm{N}$-corpos da mecânica estatística, através de técnicas estocásticas, foi um dos primeiros problemas resolvidos por Metropolis, conjuntamente com os Rosenbluth e os Teller, conduzindo ao desenvolvimento do que é actualmente conhecido por método de Monte Carlo de Metropolis. A sua cédula de nascimento é o artigo mencionado na introdução.

O neófito teve, no entanto, alguns problemas de identidade e sobrevivência. Para os enquadrar, é conveniente recordarmos a ideia fundamental do método.

\section{O método}

O objectivo essencial de método de Metropolis é estimar médias de propriedades mecânicas (energia e pressão, por exemplo) sobre um conjunto (ensemble) de configuraçōes, gerado através de um passeio aleatório no espaço configuracional dum sistema com temperatura, volume e número de moléculas constantes. O conjunto de configurações nas condições termodinâmicas referidas, designa-se por ensemble canónico. em que $E(i)$ é a energia potencial do estado $i ; \beta=1 / k T$, onde $k$ é a constante de Boltzmann e $T$ a temperatura absoluta, e $Q$ a função de partição.

Se cada configuração do ensemble for simplesmente gerada com probabilidade uniforme e a média da propriedade mecânica, $\langle f\rangle$, for realizada afectando $\mathrm{f}(\mathrm{i})$ do peso do factor de Boltzmann, $\exp [-\beta E(i)]$, conforme a equação anterior sugere, então o método será ineficiente. De facto, um número substancial de configurações terá moléculas sobrepostas implicando uma elevada energia potencial, ou seja um factor de Boltzmann muito pequeno. A sua contribuição será, então, irrelevante para a média canónica da propriedade.

A ideia genial de Metropolis, e colaboradores, foi substituir o método anterior, sugerido pela aplicação directa da equação (1), por um processo totalmente equivalente, mas operacionalmente eficiente: as configurações são geradas com probabilidade proporcional ao factor de Boltzmann e pesadas uniformemente em vez de, como a equação (1) sugere, gerar as configurações uniformemente e pesá-las com o factor de Boltzmann. Basta que se garanta a convergência estocástica do processo, isto é, que cada configuração no ensemble ocorra com uma frequência proporcional ao factor de Boltzmann.

Uma vez lavrada a prescrição é necessário aviar a receita. Metropolis et al.[1] 
executaram o passeio aleatório através da realização de uma cadeia de passos sucessivos, designada por cadeia de Markov [13,14], a qual é completamente especificada pela respectiva matriz de probabilidades de transição, cujo elemento genérico, $p(i, j)$, é a probabilidade condicional do sistema, estando no estado i no passo $n$ do processo, transitar para o estado j no passo $(n+1)$. Então, a estimativa de Monte Carlo da média da propriedade f, será:

$$
\bar{f}^{(m)}=(1 / m) \sum_{n=1}^{m} f(i(n))
$$

(2)

realizada sobre um número suficientemente grande de $m$ passos, sendo $i(n) o$ estado do sistema no passo $n$ do passeio aleatório.

Uma condição suficiente para que, com $\mathrm{o}$ aumento de $\mathrm{m}$, a estimativa anterior, $f(m)$, conviria estocasticamente para a média canónica $\langle\mathrm{f}\rangle$, é que a probabilidade de transição $p(i, j)$ satisfaça a condição de reversibilidade microscópica:

$\exp [-\beta E(i)] p(i, j)=\exp [-\beta E(j)] p(j, i)$

Num artigo recente [15] mostrámos que a equação anterior significa que, no limite da convergência, o número de estados i que transitam para o estado j é igual ao número de estados j que transitam para o estado i. Isto é, estabelece-se um equilíbrio dinâmico semelhante ao que se verifica nos processos cinéticos reversiveis de $1 .^{\text {a }}$ ordem, em que a velocidade da reacção directa iguala a da reacção inversa.

A implementação prática do processo é realizada considerando:

$$
p(i, j)=\alpha(i, j) q(i, j)
$$

onde $a(i, j)$ é uma matriz estocástica simétrica e normalizada:

$$
\sum_{j=1}^{M} \alpha(i, j)=1, \quad i=1,2, \ldots, M
$$

designada por matriz subjacente da cadeia, e

$$
\begin{array}{r}
q(i, j)=1 \text { se } E(j) \leq E(i) \quad(7) \\
q(i, j)=\exp [-\beta(E(j)-E(i))]=\exp (-\beta \Delta E) \\
\text { se } E(j)>E(i)
\end{array}
$$

(8)

A condição de normalização da matriz estocástica da cadeia de Markov é satisfeita impondo que: a probabilidade do sistema, estando no estado i no passo $n$ do processo, "transitar" para o mesmo estado i, no passo $(n+1)$, é:

$$
p(i, i)=1-\sum_{j \neq i} p(i, j)
$$

$\mathrm{Na}$ implementação usual do método, a matriz sujacente $\alpha(i, j)$ corresponde a seleccionar, com probabilidade uniforme, um estado j a partir de um número, $\mathrm{N}_{\mathrm{v}}$, de estados vizinhos do estado i. Assim, $\alpha(i, j)=1 / N_{v}$ e a equação (7) traduz uma transição incondicional para o estado j se a energia decrescer ou se mantiver. Se a energia aumentar, o estado j será aceite com probabilidade inferior à unidade, $\exp (-\beta \Delta E)$, de acordo com a equação (8). Se o estado j for rejeitado, então, a condição de normalização (9) impõe que se reconte o estado i como um novo elemento do ensemble, isto é, o acontecimento $i(n+1)=i(n)$ com probabilidade condicional 1- $\exp (-\beta \Delta \mathrm{E})$.

É de salientar que o método de Metropolis apenas faz a amostragem do espaço configuracional. As contribuições do espaço das velocidades são calculadas analíticamente e adicionadas no final dos cálculos. Isto contrasta, como veremos, com o método da dinâmica molecular, e algumas variantes do método de Monte Carlo, onde ambos os espaços são amostrados simultâneamente.

Finalmente, é importante destacar um aspecto fundamental do método. A função de partição, Q, da expressão (1) contem toda a informação sobre o sistema, de acordo com a mecânica estatística. Assim, o ideal seria calcular essa função e, a partir dela, obter todas as propriedades do sistema. Ora este é o grande problema operacional da mecânica estatistica. De facto, aparte alguns casos triviais (gás e cristal perfeitos) e o brilhante "tour de force" de Onsager sobre o modelo bidimensional de Ising [16], o cálculo da função de partição é impraticável para a maioria dos sistemas. Repare-se, no entanto, que a probabilidade de transição $\exp (-\beta \Delta \mathrm{E})=$ $\exp [-\beta E(j)] / \exp [-\beta E(i)]$, na equação (8) do algoritmo de Metropolis, evita o cálculo da função de partição. Na verdade, ela é simplesmente eliminada quando se faz a razão das probabilidades dos estados j e i respectivamente, uma vez que a função de partição não é mais do que o factor de normalização da distribuição de Boltzmann.

\section{Os problemas}

A justificação do método de Monte Carlo, apresentada na secção anterior, é a que consta em alguns livros de texto actuais $[17,18]$ e foi despida de ambiguidades ao longo dos últimos cinquenta anos. Mas quem leia a cédula de nascimento do método, isto é, o artigo de Metropolis et al. [1] já mencionado, aperceber-se-à de que as coisas não estavam tão bem fundamentadas nessa época. É uma característica típica das grandes realizações científicas. Que, infelizmente, nem sempre destacamos na nossa actividade docente.

Não é de estranhar, então, que pouco depois da publicação do método em Junho de 1953, tenha entrado em cena John Kirkwood, uma das respeitáveis autoridades em mecânica estatística, sugerindo que o método estava errado! É interessante analisar as suas objeções e as razões intrínsecas do seu cepticismo.

Kirkwood era consultor do Laboratório de Los Alamos e teve conhecimento dos trabalhos de Metropolis e colaboradores antes da referida publicação. De início, apercebeu-se das grandes potencialidades do método e estimulou jovens doutorados sob a sua orientação (como W.W. Wood) a integrarem essas originais investigações.

0 artigo de Metropolis et al. [1], de 1953, simulou a equação de estado dum sistema de discos rígidos comparando-a com as previsões da teoria do 
volume livre e com a expansão do virial. $\mathrm{O}$ acordo obtido foi excelente. Entretanto, Marshall e Arianna Rosenbluth aplicavam o método ao estudo da equação de estado das esferas rígidas cujo artigo veio a ser publicado em 1954 [19]. O objectivo principal era analisar o problema das transições de fase e comparar os resultados das simulações com a teoria do volume livre, a expansão do virial e com a então recente teoria da sobreposição de Kirkwood e Born-Green-Yvon [20]. Esta última, constituia um significativo avanço na teoria dos líquidos e vários estudantes de Kirkwood tinham dedicado grande parte das suas teses de doutoramento à resolução numérica da equação teórica, o que não era trivial com o equipamento computacional da época. Mas o trabalho dos Rosenbluth disparou uma seta ao coração da teoria de Kirkwood quando concluiu: "The [simulated] equation of state for three-dimensional hard spheres casts doubt on the adequacy of the superposition approximation at high densities". É fácil compreender que o entusiasmo inicial de Kirkwood sobre o método de Monte Carlo tenha diminuído e que tenha informado Marshall Rosenbluth, em Novembro de 1953, que estava convencido de que o método de Monte Carlo estava errado [4]. Um ar gelado desceu sobre Los Alamos! Mas vejamos os argumentos de Kirkwood.

A primeira objeção referia-se ao processo de recontagem do estado i quando o estado j for rejeitado, o qual referimos na secção anterior. Na opinião de Kirkwood o estado i(n) não deveria ser recontado, mas novos estados deveriam ser gerados até que um fosse aceite. $M$. Rosenbluth fez uma nova demonstração do método e, juntamente com W.W. Wood, estudou sistemas com soluções analíticas exactas para as comparar com os resultados das simulaçōes. Após esta intensa labuta, o grande Kirkwood manifestou-se convencido sobre a necessidade do processo de recontagem e Los Alamos rejubilou. Mas foi paz de pouca duração!

Em Janeiro de 1954, Kirkwood entra em cena novamente com outro problema. Dizia que o método dava tratamento

preferencial à transição para os estados de menor energia através das equações (7) e (8). Quanto a ele, a probabilidade $q(i, j)$ deveria ser sempre simétrica, isto é, dada pelo que ele designou como a regra de Kahan expressa pela equação(10):

$$
q(i, j)=\frac{\exp [-\beta E(j)]}{\exp [-\beta E(i)]+\exp [-\beta E(j)]}
$$

Rosenbluth e Wood concordaram que a equação (10) era válida, mas também estavam seguros que o par de equações (7) e (8) estava correcto e, intuitivamente, sentiam que era o melhor. Reiniciou-se uma intensa discussão, até que, em Abril de 1954, Wood [4] recebeu o veredicto final de Kirkwood: "I was finally able to find the time to devote a weekend to thinking about the Monte Carlo problem". Continuou, dizendo que admitia o par de equações (7) e (8) como "one of the correct ones". Mas, rematou: "Marshall's arguments were at no time helpful and your modes of exposition were opaque"!

A partir desse momento, Kirkwood foi um activo promotor do método de Monte Carlo dando-o a conhecer nos seus importantes contactos científicos. Que belo e estimulante testemunho de humildade científica!

É importante sublinhar que tanto o par de equaçôes (7) e (8) como a equação (10) são válidas como probabilidades de transição. Na verdade, a escolha não é única. Basta que obedeça à condição da reversibilidade microscópica como é o caso daquelas equações, o que os leitores poderão facilmente verificar. Este é um dos aspectos mais poderosos do método de Monte Carlo. A intuição do grupo de Los Alamos sobre a vantagem do par (7) e (8) está hoje bem justificada [17]. De facto, essas probabilidades de transição minimizam, em geral, a variância estatística das médias de Monte Carlo. Como tal, são as usualmente utilizadas. Mas isso não significa que, em aplicações específicas, não seja vantajoso utilizar outras probabilidades de transição.

\section{Comentários finais}

0 método de Monte Carlo sobreviveu às suas dores intestinais e é hoje uma ferramenta poderosa em mecânica estatística bem como em outras áreas teóricas e experimentais. Em 1957, Alder e Wainwright lançaram o método da dinâmica molecular, o equivalente determinístico do método de Monte Carlo. A aliança entre os dois métodos [21, 22] demonstrou, inequivocamente, a existência de uma transição de fase fluído-sólido para o sistema de esferas rígidas, um fenómeno de crucial importância para a compreensão da estrutura dos líquidos e dos sólidos. Ainda em 1957, Wood e Parker [23] aplicaram o método de Monte Carlo a um estudo detalhado do sistema de Lennard-Jones. Estas grandes realizações levaram Temperley [24] a afirmar: "A key year for liquid state physics was 1957 "

O dealbar da década de 1960 aproximava-se e nela iriam aparecer consideráveis desenvolvimentos e aplicaçōes, a par do incremento do poder computacional. 0 ciclo da adolescência da simulação computacional em mecânica estatística completar-se-ia, e ela iria definitivamente entrar, na década de 70 , na idade adulta. Mas estes aspectos ficarão para o próximo artigo onde referiremos, também, o início da simulação computacional em Portugal.

\section{Agradecimentos}

$\mathrm{O}$ autor agradece reconhecidamente ao avaliador deste artigo as sugestões relativas a um maior destaque das contribuiçōes de John von Neumann e ao facto do algoritmo de Metropolis evitar o cálculo da função de partição.

\section{Referências}

[1] N. Metropolis, A. Rosenbluth, M. Rosenbluth, A. Teller, E. Teller, J. Chem. Phys., 21, 1087 (1953)

[2] Web site do Laboratório Nacional de Los Alamos: www.lanl.gov/worldview 
[3] E. Segrè, From X-rays to Quarks, W.H. Freeman, San Francisco, Cal.,1980.

[4] W.W. Wood, em Proceedings of the International School of Physics "Enrico Fermi", North-Holland, pág. 3, 1986.

[5] N. Metropolis, S. Ulam, J. Am. Stat. Assoc., 44, 335 (1949).

[6] J. von Neumann, Mathematical Foundations of Quantum Mechanics, Princeton University Press, 1955

[7] E. Regis, Who Got Einstein's Office?, Penguin Books, Londres, 1987.

[8] J. Halton, SIAM Rev., 12, 1 (1970).

[9] J. von Neumann, NBS Appl. Math. Series, 12, 36 (1951).

[10] J. von Neumann, Bull. Amer. Math. Soc., 51, 660 (1945)
[11] J.M. Hammersley, D.C. Handscomb, Monte Carlo Methods, Chapman and Hall, London, 1964.

[12] Lord Kelvin, Philos. Mag., (6)2, 1 (1901). [13] W. Feller, An Introduction to Probability Theory and its Applications, Vol. I, 3. ${ }^{a}$ edição, John Wiley \& Sons, 1970.

[14] G.G. Lowry, J.M. Myhre, M. Fluendy, em Markov Chains and Monte Carlo Calculations in Polymer Science, ed. G.G. Lowry, Marcel Dekker,Inc., 1970.

[15] F.M.S.S. Fernandes, Rev. Ciência, Série VII, 2, 31 (2001).

[16] L. Onsager, Phys. Rev., 65, 117 (1944).

[17] M.P. Allen, D.J. Tildesley, Computer Simulation of Liquids, Claredon Press, Oxford, 1987.
[18] D. Frenkel, B. Smit, Understanding Molecular Simulation. From Algorithms to Applications, 2. ${ }^{a}$ Edição, Academic Press, 2002.

[19] M. Rosenbluth, A. Rosenbluth, J. Chem. Phys., 22, 881 (1954).

[20] J. Kirkwood, E.K. Maun, B.J. Alder, J. Chem. Phys. 18, 1040 (1950).

[21] B.J. Alder, T.E. Wainwright, J. Chem. Phys., 27, 1208, (1957).

[22] W.W.Wood, J.D. Jacobson, J. Chem. Phys., 27, 1207, (1957).

[23] W.W.Wood, F.R. Parker, J. Chem. Phys., 27, 720, (1957).

[24] H.N.V. Temperley, J.S. Rowlinson, G.S. Rushbrooke, Physics of Simple Liquids, pág. 7, Wiley, New York, 1968.

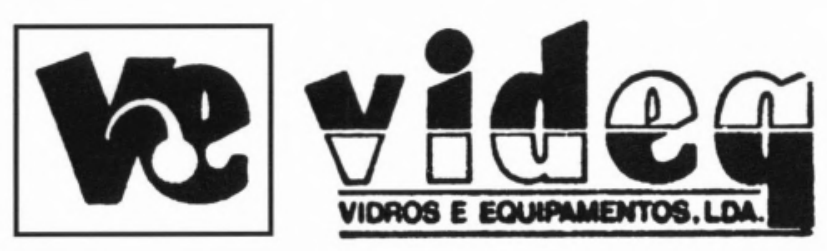

Equipamento de Laboratório Balanças - Centrifugas - Aparelhos de $\mathrm{pH}$ - Tituladores

Condutímetros - Agitadores - Espectrofotómetros Microscópios - etc.

Vidros e Plásticos de Laboratório Distribuidores NORMAX

Material Didáctico

Ensino Secundário e Superior

Representantes exclusivos SISTEDUC - Sistemas Educativos S.A.

Rua Soeiro Pereira Gomes, 15 r/c Frente

Bom Sucesso - 2615 Alverca

Telefs. (01) 95704 20/1/2 - Fax (351-1-957 04 23) - Portugal 


\section{Subscribe Now!}

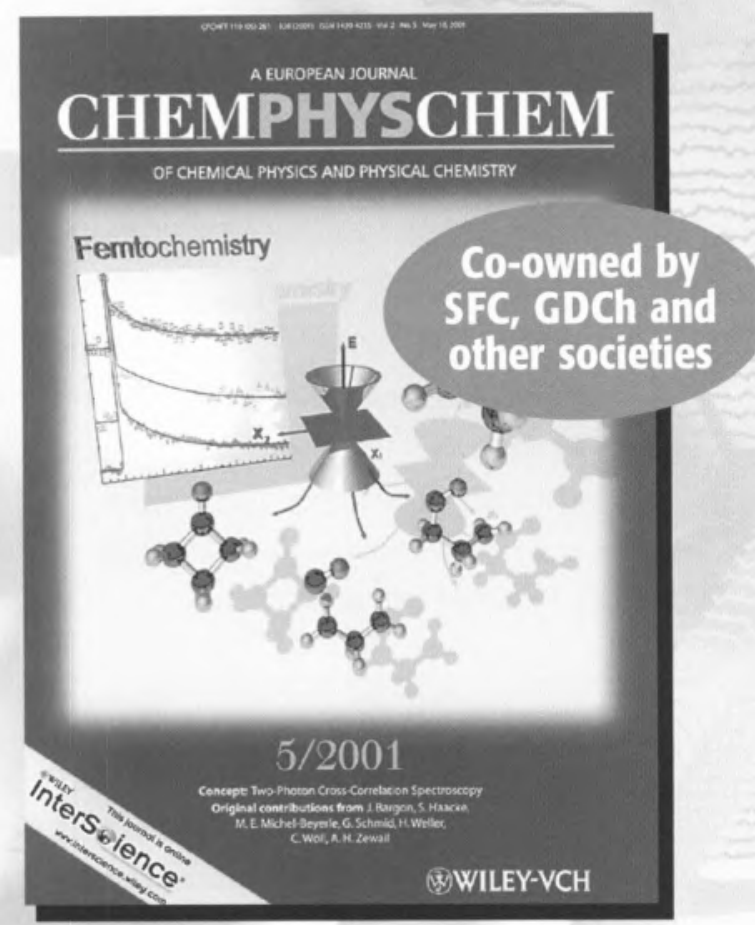

\section{ChemPhysChem}

A European Journal of Chemical Physics and Physical Chemistry

2002 Volume 3, 12 issues per year,

ISSN Print 1439-4235

ISSN Electronic 1439-7641
ChemPhysChem - Where Chemistry Meets Physics Meets Chemistry...

ChemPhysChem amalgamates the wide and flourishing field ranging - to name just a few topics -

- from atmospheric science to hard and soft condensed matter

- from femtochemistry to nanotechnology

- from complex biological systems to single molecule research

- from clusters and colloids to catalysis and surface science

- from electro- to photochemistry

\section{Papers from distinguished scientists worldwide, such as}
Z. I. Alferov
G. Ertl
C. A. Mirkin
C. Amatore
C. Friend
C. N. R. Rao
C. D. Bain
J. S. Kilby
V. Balzani
H. Kroemer
J.-M. Savéant
C. Bräuchle
R. Lavery
R. J. Saykally
E. A. Carter
J.-M. Lehn
G. Wegner
A. Corma
R. D. Levine
C. Zannoni
F. C. De Schryver
H. Matsuhara
R. N. Zare
A. H. Zewail

Available as a separate journal and as a part of attractive packages with Angewandte Chemie (Int. Ed.) Please visit www.chemphyschem.com

\section{Virtual Sample Copy: FREE online access to full text of sample copy: www.interscience.wiley.com}

Please enter my/our 2002 subscription to ChemPhysChem

Please tick: $\square$ private $\square$ business

At the institutional rate*:

print
$\in$ electronic
sFr 1028,- $\quad \square \in$ SFr 1028,-
US\$ 648,- $\square$ US\$ 648,-
or a 5\% premium, institutions can also
hoose both print and online access.

At the personal member rate: print

$\square € \quad 148,-$ sFr 248,US\$ 158,-

Europe Switzerland All other countries

Name

Address

City/Postcode

\section{Please send me a free sample copy}

Please return this order form to your local bookseller or to:

Customers in Germany, Austria, and Switzerland:

WILEY-VCH Reader Service

P. O. Box 1011 61,

D.69451 Weinheim, Germany

Phone: +49 (0) $6201-60614$

Fax: $+49(0) 6201-606172$
e-mail: subservice@wiley-vch.de
Customers in all other areas: John Wiley \& Sons, Ltd. Journals Administration Department 1 Oldlands Way Bognor Regis West Sussex, PO22 95A, UK Phone: $+44(0)$ 1243-779 777 Fax: +44 (0) 1243-843232 e-mail: cs-journals@wiley.co.uk 